\title{
Motional Coherence in Fluid Phospholipid Membranes
}

\author{
Maikel C. Rheinstädter ${ }^{1,2}$. ${ }^{\text {. Jhuma Das }}{ }^{1}$, Elijah J. Flenner ${ }^{1}$, Beate Brüning ${ }^{2,3}$, Tilo Seydel ${ }^{2}$, and Ioan Kosztin ${ }^{1}$ \\ ${ }^{1}$ Department of Physics and Astronomy, University of Missouri-Columbia, Columbia, MO 65211, U.S.A. \\ ${ }^{2}$ Institut Laue-Langevin, 6 rue Jules Horowitz, B.P. 156, 38042 Grenoble Cedex 9, France and \\ ${ }^{3}$ Institut für Röntgenphysik, Friedrich-Hund Platz 1, 37077 Göttingen, Germany
}

(Dated: March 4, 2022)

\begin{abstract}
We report a high energy-resolution neutron backscattering study, combined with in-situ diffraction, to investigate slow molecular motions on nanosecond time scales in the fluid phase of phospholipid bilayers of 1,2-dimyristoyl-sn-glycero-3-phoshatidylcholine (DMPC) and DMPC/40\% cholesterol (wt/wt). A cooperative structural relaxation process was observed. From the in-plane scattering vector dependence of the relaxation rates in hydrogenated and deuterated samples, combined with results from a $0.1 \mu \mathrm{s}$ long all atom molecular dynamics simulation, it is concluded that correlated dynamics in lipid membranes occurs over several lipid distances, spanning a time interval from pico- to nanoseconds.
\end{abstract}

PACS numbers: 87.16.dj, 87.14.Cc, 83.85.Hf, 87.15.ap, 83.10.Mj

It is speculated that atomic and molecular motions in regions of biomolecular systems with strong local interactions are highly correlated on certain range of time and length scales [1, 2, In proteins intra-protein correlations are believed to be essential for their biological functioning, such as protein folding, domain motion and conformational changes. Very recently, inter-protein correlations in protein crystals and also membranes have been reported from experiment and simulation [3, 4. Experimental and computational effort has been invested to study collective molecular motions in phospholipid model membranes [5, 6, 7, 8, to understand the possible impact on physiological and biological functions of the bilayers, such as transport processes [9], and eventually their implication on function of membrane-embedded proteins. While fast (picosecond) propagating collective microscopic fluctuations in the plane of the bilayer can be understood as sound waves [10, 11, the slow (nanosecond) in-plane mesoscopic fluctuations (undulations) are governed by the elasticity properties of the bilayers [12]

We studied dynamical modes at nearest neighbor distances of the lipid molecules using the neutron backscattering technique [13]. These modes are too fast to be accessed by x-ray photon correlation spectroscopy and the lateral length scales involved are too small to be resolved by dynamic light scattering or the neutron spinecho technique. Selective deuteration was used to discriminate relaxations due to collective molecular motions from relaxations arising from localized, single molecule excitations. In this Letter, we examine results of inelastic neutron scattering experiments that demonstrate the existence of slow coherent motion of lipid molecules in the fluid phase of phospholipid bilayers. From the inplane scattering vector dependence $\left(q_{\|}\right)$of the measured relaxation rates, combined with results of a $0.1 \mu \mathrm{s}$ long all atom molecular dynamics (MD) simulation, we find that the cooperative structural dynamics in lipid membranes occurs over several lipid distances, spanning a time inter- val from pico- to nanoseconds.

The experiments were carried out at the cold neutron backscattering spectrometer IN16 [14 at the Institut Laue-Langevin (ILL) with an energy resolution of about $0.9 \mu \mathrm{eV}$ FWHM $(\lambda=6.27 \AA)$. An energy transfer of $-15 \mu \mathrm{eV}<E<+15 \mu \mathrm{eV}$ and a $q$ range of $0.43 \AA^{-1}<q<1.92 \AA^{-1}$ were scanned, accessing time scales of $0.28 \mathrm{~ns}<\mathrm{t}<4.6 \mathrm{~ns}$ and length scales of $3.2 \AA<\mathrm{d}<14.6 \AA$. A separate line of diffraction detectors allowed to determine the bilayer structure in-situ. Protonated (DMPC-h) and partially (acyl chain) deuterated 1,2-dimyristoyl-sn-glycero-3-phoshatidylcholine (DMPCd54), along with protonated cholesterol were obtained from Avanti Polar Lipids. Highly oriented multi lamellar membrane stacks of several thousands of lipid bilayers of DMPC and DMPC/cholesterol were prepared by spreading a solution of typically $25 \mathrm{mg} / \mathrm{ml}$ lipid and lipid/40\% cholesterol (wt/wt) in trifluoroethylene/chloroform (1:1) on 2" silicon wafers, followed by subsequent drying in vacuum and hydration from $\mathrm{D}_{2} \mathrm{O}$ or $\mathrm{H}_{2} \mathrm{O}$ vapor. Twenty such wafers (five for DMPC-h) separated by small air gaps were combined and aligned with respect to each other to create a "sandwich sample" consisting of several thousands of highly oriented lipid bilayers (total mosaicity about $0.5^{\circ}$ ). The deuterated (protonated) samples had a total mass of about $400 \mathrm{mg}(100 \mathrm{mg})$ of lipid or lipid/cholesterol. The samples were mounted in a hermetically sealed aluminum container within a cryostat and hydrated from $\mathrm{D}_{2} \mathrm{O}$ or $\mathrm{H}_{2} \mathrm{O}$ vapor. By aligning the bilayer normal at 135 , respective 45 degrees with respect to the incoming neutron beam, the momentum transfer could be placed in the plane of the bilayers $\left(q_{||}\right)$or perpendicular to the membranes $\left(q_{z}\right)$. The lamellar spacing of the DMPC sample was determined to $d_{z}=54 \AA$ at $\mathrm{T}=30^{\circ} \mathrm{C}$, which corresponds to a relative humidity of $\mathrm{RH}=99.6 \%$ [15]. Five different samples (referred to as $\mathrm{S} 1, \ldots, \mathrm{S} 5)$ were prepared: 


\begin{tabular}{c|l|c|c} 
Sample & Bilayer & Cholesterol Content & Hydration \\
\hline S1 & DMPC -d54 & - & $\mathrm{D}_{2} \mathrm{O}$ \\
S2 & DMPC -d54 & - & $\mathrm{H}_{2} \mathrm{O}$ \\
S3 & DMPC -h & - & $\mathrm{D}_{2} \mathrm{O}$ \\
S4 & DMPC -h & - & $\mathrm{H}_{2} \mathrm{O}$ \\
S5 & DMPC -d54 & $40 \%$ cholesterol & $\mathrm{D}_{2} \mathrm{O}$
\end{tabular}

Neutrons are scattered by the atomic nuclei, and each element intrinsically has non zero coherent and incoherent scattering cross sections. In experiments the different contributions can be enhanced with respect to each other, by, e.g., using different isotopes or selective deuteration. While in protonated samples the incoherent scattering is dominant and the time-autocorrelation function of individual scatterers is accessed, (partial) deuteration emphasizes the coherent scattering and probes the pair correlation function. By careful analysis of the scattering of all samples S1 to S5, it was possible to emphasize between the collective and self motions in the underlying dynamics. Sample S5 allowed us to investigate the effect of cholesterol incorporation on the collective dynamics of the lipid backbone because protonated cholesterol was used.

Figure 1(a) depicts in-plane diffraction patterns, i.e., structure factors $S\left(q_{\|}\right)$, at $303 \mathrm{~K}$. Subtracting $S\left(q_{||}\right)$for samples S3 and S1 emphasizes the coherent scattering of the lipid acyl chains. Similarly, subtraction of $S\left(q_{\|}\right)$for S3 and S5 emphasizes the coherent lipid tail scattering in the cholesterol sample. Finally, to emphasize coherent water scattering, the diffraction pattern of S4 was subtracted from that of S3. There are two peaks in $S\left(q_{\| \mid}\right)$ of the lipid chains around $q_{\|}=0.7 \AA^{-1}$ and $1.4 \AA^{-1}$, which correspond to the nearest neighbor distances of phospholipid head groups and the acyl chains in DMPC, respectively. The peaks shift to smaller $q_{||}$values in the presence of cholesterol, indicating a slight increase in the nearest neighbor distances. The water scattering is enhanced towards the water correlation peak around $2 \AA^{-1}$, but there is no pronounced diffraction peak within the instrumental resolution.

Figure 2 exemplarily depicts spectra measured at $q_{||}=$ $0.5 \AA^{-1}$ and $1.5 \AA^{-1}$ for $\mathrm{S} 1$ and $\mathrm{S} 4$ at a temperature of $\mathrm{T}=303 \mathrm{~K}$, in the fluid phase of the bilayers. A fast and a slow relaxation process were visible in the data and data were therefore fitted using two Lorentzians and convoluted with the instrumental resolution. Because the faster process (the broad Lorentzian) could not be determined with sufficient accuracy, this Letter focuses on the slower relaxation process $\left(\omega_{1}\right)$. $\omega_{1}$ might contain contributions from incoherent and coherent scattering. A quantitative determination of the coherent and incoherent relaxation rates would require purely coherent and purely incoherent scatterers or neutron polarization analysis (which has not yet been realized on a neutron backscattering spectrometer) to discriminate the different contributions to $\omega_{1}$. A convolution of incoher-

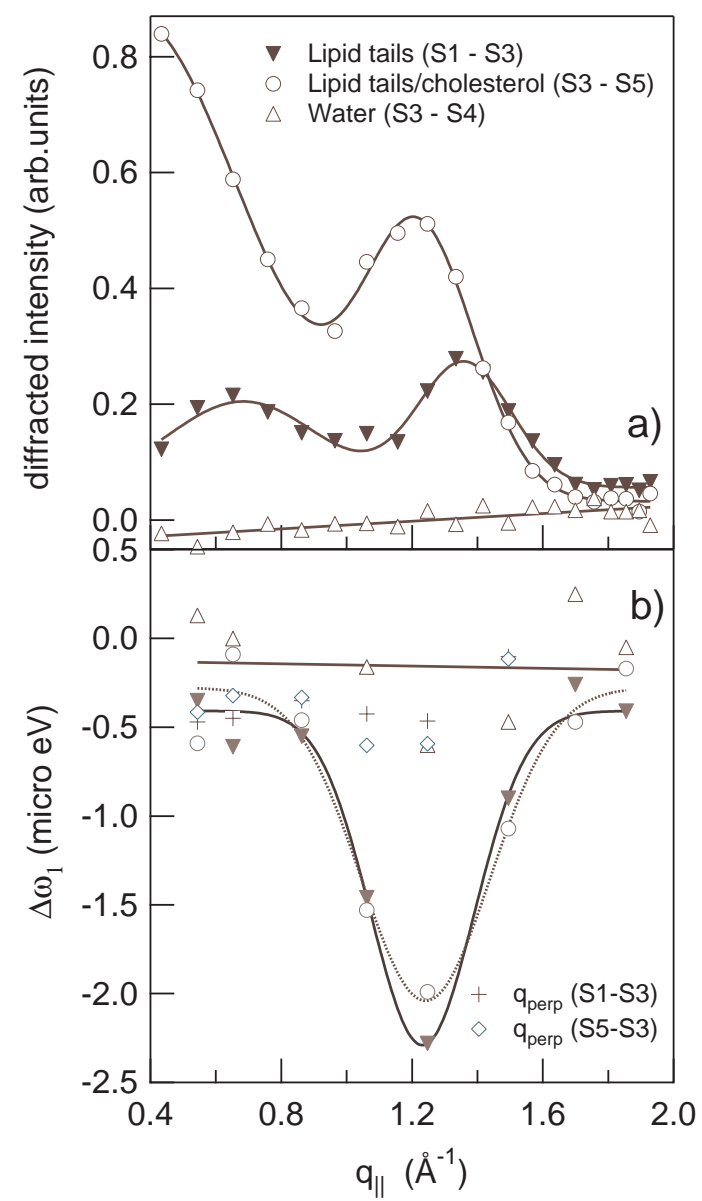

FIG. 1: (Color online). (a) Diffraction patterns generated by subtraction of $S\left(q_{\|}\right)$for pairs of samples $\mathrm{S} 1, \ldots, \mathrm{S} 5$, which emphasize contributions from collective molecular motions of the lipid acyl chains, and the water molecules (see text for explanation). (b) $\Delta \omega_{1}$ for the lipid acyl chains, the membrane hydration water, and the lipid chains 'distorted' by incorporating $40 \%$ cholesterol. For the water, the difference of $\Delta \omega_{1}$ of samples S1 and S2 was taken.

ent and coherent relaxation processes, each described by a Lorentzian peak shape (implicating a single exponential relaxation process), was assumed. Since the convolution of two Lorentzians with widths $\Delta \omega_{1}^{\prime}$ and $\Delta \omega_{1}^{\prime \prime}$ is again a Lorentzian peak shape with a HWHM of $\Delta \omega_{1}=\Delta \omega_{1}^{\prime}+\Delta \omega_{1}^{\prime \prime}$, by subtracting the Lorentzian widths of spectra of protonated (mainly incoherent scatterers) from the (partially) deuterated samples (mainly coherent scatterers), it was qualitatively possible to distinguish the cooperative dynamics relative from the single-particle dynamics.

Fig. 1. (b) shows the difference in the quasielastic width $\Delta \omega_{1}\left(q_{\|}\right)$for the slow process for the lipid acyl chains, the membrane hydration water, and the lipid chains 'distorted' by incorporating $40 \%$ cholesterol. So does, e.g., subtracting the widths of spectra S1-S3 emphasize the 


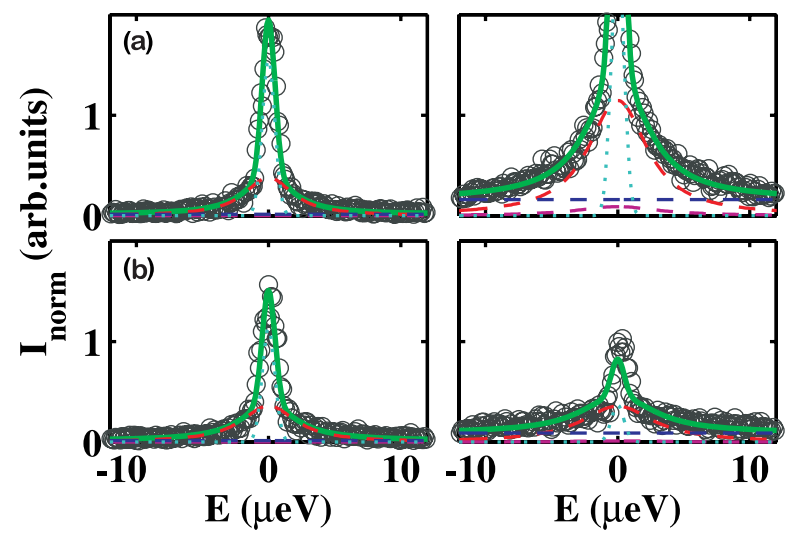

FIG. 2: (Color online). Exemplary inelastic spectra in the fluid phase of the bilayers. Left column: $q_{||}=0.5 \AA^{-1}$; right column: $q_{\|}=1.5 \AA^{-1}$. a) DMPC-d54/D $2 \mathrm{O}$ (Sample S1), b) DMPC-h $/ \mathrm{H}_{2} \mathrm{O}$ (Sample S4). Data have been fitted using the instrumental resolution (dotted), two Lorentzian peak shapes (dashed), and a constant background (dashed). The total fit is shown as solid line.

coherent lipid tail dynamics, analogous to the diffraction discussed above. $\Delta \omega_{1}\left(q_{\|}\right)$shows a pronounced minimum around $q_{||}=1.22 \AA^{-1}$ for the lipids and lipids/cholesterol sample and qualitatively reproduces the behavior of $S\left(q_{\|}\right)$in Fig. 1(a). This points to a strong coupling of dynamical properties to the static structure of the system and to a softening of the coherent relaxation rate around the maximum of the static structure factor $S\left(q_{\|}\right)$. The slowing down of the decay of the density autocorrelation function for distances corresponding to nearest neighbors is known as de Gennes narrowing [16. The momentum dependence of the dynamical parameters in the collective dynamics case is not trivial as in the single-particle case but points to coherent structural relaxation. Because the out-of-plane component $\left(q_{\perp}\right)$ of the scattering vector (also in Fig. 1(b)) shows no minimum, it is concluded that the relaxation process is confined to the plane of the membranes.

To gain further insight in the above relaxation process, we performed an all atom MD simulation of DMPC and analyzed the motion of the carbons within the lipid tails. The fully solvated system, built from a pre-equilibrated DMPC bilayer [17, contained 128 lipid and 2577 TIP3 water molecules. The MD simulation was performed with NAMD-2.6 [18] using the CHARMM27 [19] force field. The pair interactions were turned off smoothly from $10 \AA$ to $12 \AA$, and the long-range electrostatic interactions were calculated using the PME method [20. The simulation was performed in the NVT ensemble using periodic boundary conditions. A constant temperature of $\mathrm{T}=303 \mathrm{~K}$ was maintained by employing a Langevin thermostat with a coupling constant of $0.05 \mathrm{ps}^{-1}$. After proper energy minimization and thermal equilibration, a production run of $0.1 \mu$ s was carried out on $40 \mathrm{CPUs}$

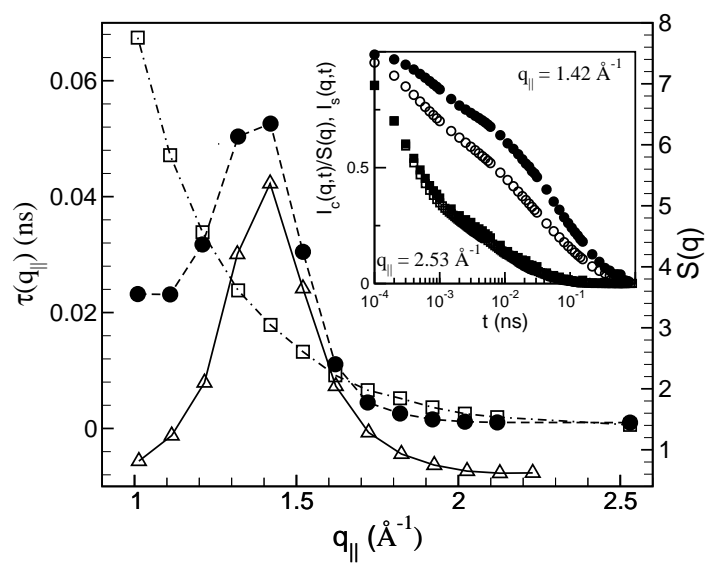

FIG. 3: The slow relaxation times $\tau_{c}$ (closed circles) and $\tau_{s}$ (open squares) as a function of wave-vector $q_{\|}$. Also shown is the static structure factor $S\left(q_{||}\right)$(open triangles). Inset: $I_{s}(q, t)$ (open symbols) and $I_{c}(q, t)$ (filled symbols) for $q_{\|}=$ $2.53 \AA^{-1}$ (lower curves) and $1.42 \AA^{-1}$ (upper curves).

of a dual core $2.8 \mathrm{GHz}$ Intel Xeon EM64T cluster with a performance of 0.2 day/ns.

To examine the structural relaxation of the lipid tails we calculated the in-plane incoherent (self) $I_{s}\left(q_{\|}, t\right)=$ $(1 / N) \sum_{n}\left\langle e^{-i \vec{q}_{||} \cdot\left[\vec{r}_{n}(0)-\vec{r}_{n}(t)\right]}\right\rangle$ and the coherent $I_{c}\left(q_{||}, t\right)=$ $(1 / N) \sum_{n} \sum_{m}\left\langle e^{-i \vec{q}_{||}\left[\vec{r}_{n}(0)-\vec{r}_{m}(t)\right]}\right\rangle$ intermediate scattering functions. The sums are taken over the $N$ carbon atoms in the lipid tails and $\vec{r}_{n}(t)$ is the position of the $n^{t h}$ atom at time $t$. The scattering vector $\vec{q}_{\|}$is parallel to the $x-y$ plane of the lipid bilayer. By examining $I_{s / c}\left(q_{\|}, t\right)$ we can isolate purely coherent and incoherent contributions to the scattering due to the carbons within the lipid tails.

The minimum in $\Delta \omega_{1}\left(q_{||}\right)$shown in Fig. 1 suggests a longer relaxation time for $I_{c}\left(q_{\|}, t\right)$ than for $I_{s}\left(q_{\|}, t\right)$ for $q_{\|}$values around the peak of $S\left(q_{\|}\right)=I_{c}\left(q_{\|}, 0\right)$. Shown in inset to Fig. 3 are $I_{s / c}\left(q_{\|}, t\right)$ for $q_{\|}=2.5 \AA^{-1}$ and $1.42 \AA^{-1}$. For $q_{\|}=2.5 \AA^{-1}$ the decay times for $I_{s}\left(q_{\|}, t\right)$ and $I_{c}\left(q_{\|}, t\right)$ are almost identical, but $I_{c}\left(q_{\|}, t\right)$ decays slower for $q_{\|}$around the first peak of the static structure factor. We defined the decay time $\tau\left(q_{\|}\right)$as when $I_{s / c}\left(q_{\|}, \tau\left(q_{\|}\right)\right) / I_{s / c}(q, 0)=e^{-1}$. Shown in Fig. 3 are $\tau_{s}\left(q_{\|}\right)$and $\tau_{c}\left(q_{\|}\right)$along with $S\left(q_{\|}\right)$. Note that the nonmonotonic behavior of $\tau_{c}\left(q_{||}\right)$yields a minimum in the difference $\tau_{s}-\tau_{c}$ around the peak of $S\left(q_{\|}\right)$. Thus, the MD simulation results are in qualitative agreement with those from the experiment and reinforces the conclusion that the difference of the quasielastic widths $\Delta \omega_{1}\left(q_{\| \mid}\right)$is due to correlated dynamics of the lipid acyl chains.

To determine the temporal and spatial extent of the correlated motion of the lipid tails, we calculated the correlation function

$$
g_{\Delta}(r, t)=\frac{V}{\langle\Delta r\rangle^{2} N} \times
$$




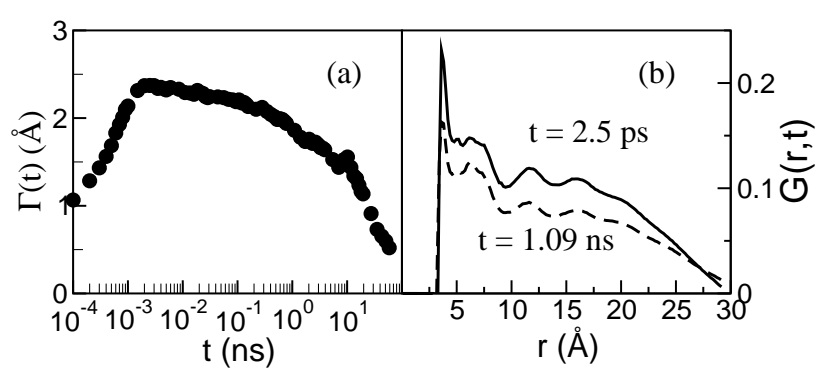

FIG. 4: Measures of the correlated dynamics $\Gamma(t)=$ $\int G(r, t) \mathrm{d} r . \quad \Gamma(t)$ is a measure of the size of the correlated displacement at a time $t$, and $G(r, t)$ measures the spatial range of the correlations.

$$
\sum_{n, m}^{\prime}\left\langle\Delta r_{n}(t) \Delta r_{m}(t) \delta\left(r-\left|\vec{r}_{n}(0)-\vec{r}_{m}(0)\right|\right)\right\rangle
$$

where $\Delta r_{n}(t)=\left|\vec{r}_{n,||}(t)-\vec{r}_{n,||}(0)\right|$ represents the displacement of carbon atoms in the $x-y$ plane of the membrane, and the prime denotes a restricted double sum in which carbon pairs belonging to the same lipid tail are excluded. In the absence of displacement correlations, $g_{\Delta}(r, t)=g_{1}(r)=\left(V / N^{2}\right)\left\langle\sum_{n, m}{ }^{\prime} \delta\left(r-\left|\vec{r}_{n}-\vec{r}_{m}\right|\right)\right\rangle$. Thus $\Gamma(t)=\int\left[g_{\Delta}(r, t) / g_{1}(r)-1\right] \mathrm{d} r$ provides a measure of the correlation of in plane displacements of the carbon atoms within the lipid tails between different lipids [21].

Examination of $\Gamma(t)$, Fig. 4(a), shows that the displacements of the lipid acyl tails are correlated for times from around 1 ps to $1 \mathrm{~ns}$, i.e., for much longer than the microscopic collision time but shorter than the time needed for a lipid to diffuse one lipid diameter. This is exactly the time window of the structural relaxation probed by the neutron scattering experiments and corresponds to the decay times $\tau_{s / c}$. Thus, we associate $\Delta \omega_{1}$ observed in the experiments and the difference in decay times $\tau_{c}-\tau_{s}$ to correlated motion of lipid tails.

Finally, the spatial decay of $G(r, t)=g_{\Delta}(r, t) / g_{1}(r)-1$ yields the length scale associated with the correlated displacements. Shown in Fig. $4 \mathrm{p}$ is $G(r, t)$ for $t_{1}=2.5$ ps (at the peak of $\Gamma(t))$ and $t_{2}=1.1 \mathrm{~ns}$. For both times $G(r, t)$ does not decay to zero until around $30 \AA$. Therefore, the lipid tails displacements are correlated for at least four to six lipid diameters between $t_{1}$ and $t_{2}$.

In conclusion we found experimental evidence for a cooperative structural relaxation process in fluid phospholipid membranes. An all atom molecular dynamics simulations demonstrated that the displacements of the lipid tails are correlated for up to six lipid diameters for a time span from picoseconds to around a nanosecond. Experimental data of the sample containing cholesterol proved that the process is present not only in pure lipid bilayers, but also in more complex and relevant membranes. A possible implication of this motional coherence of the lipid acyl chains is, e.g., that information about a lo- cal structural perturbance of the lipids could propagate in the bilayer, which might be relevant for the understanding of processes and functions involving collective structural changes.

We acknowledge financial support from the DFG through project SA 772/8-2. We thank T. Salditt (Göttingen) for continuous support, E. Kats for critical reading of the manuscript and valuable comments, and the ILL for the allocation of beam time. Computer time was generously provided by the University of Missouri Bioinformatics Consortium.

* Electronic address: RheinstadterM@missouri.edu

[1] C. L. Brooks, M. Karplus, and B. M. Pettit, Proteins: A theoretical perspective of dynamics, structure and thermodynamics (J. Wiley \& Sons, New York, 1989).

[2] R. Lipowsky and E. Sackmann, eds., Structure and Dynamics of Membranes, vol. 1 of Handbook of Biological Physics (Elsevier, Amsterdam, 1995).

[3] V. Kurkal-Siebert, R. Agarwal, and J. C. Smith, Phys. Rev. Lett. 100, 138102 (4 pages) (2008).

[4] M. C. Rheinstadter, K. Schmalzl, K. Wood, and D. Strauch (2008), http://arxiv.org/abs/0803.0959.

[5] T. Bayerl, Curr. Opin. Colloid Interface Sci. 5, 232 (2000).

[6] M. C. Rheinstädter, T. Seydel, W. Häußler, and T. Salditt, J. Vac. Sci. Technol. A 24, 1191 (2006).

[7] J. S. Hub, T. Salditt, M. C. Rheinstädter, and B. L. de Groot, Biophysical J. 93, 3156 (2007).

[8] M. Tarek, D. Tobias, S.-H. Chen, and M. Klein, Phys. Rev. Lett. 87, 238101 (4 pages) (2001).

[9] S. Paula, A. Volkov, A. Van Hoek, T. Haines, and D. Deamer, Biophys. J. 70, 339 (1996).

[10] S. Chen, C. Liao, H. Huang, T. Weiss, M. Bellisent-Funel, and F. Sette, Phys. Rev. Lett. 86, 740 (2001).

[11] M. C. Rheinstädter, C. Ollinger, G. Fragneto, F. Demmel, and T. Salditt, Phys. Rev. Lett. 93, 108107 (4 pages) (2004).

[12] M. C. Rheinstädter, W. Häussler, and T. Salditt, Phys. Rev. Lett. 97, 048103 (4 pages) (2006).

[13] M. C. Rheinstädter, T. Seydel, and T. Salditt, Phys. Rev. E 75, 011907 (8 pages) (2007).

[14] B. Frick and M. Gonzalez, Physica B 301, 8 (2001).

[15] N. Chu, N. Kučerka, Y. Liu, S. Tristram-Nagle, and J. F. Nagle, Phys. Rev. E 71, 041904 (8 pages) (2005).

[16] P. DeGennes, Physica (Amsterdam) 25, 825 (1959).

[17] A. A. Gurtovenko, M. Patra, M. Karttunen, and I. Vattulainen, Biophys J 86, 3461 (2004).

[18] J. Phillips, R. Braun, W. Wang, J. Gumbart, E. Tajkhorshid, E. Villa, C. Chipot, R. Skeel, L. Kale, and K. Schulten, J Comput Chem 26, 1781 (2005).

[19] S. Feller and J. MacKerell, J. Phys. Chem. B 104, 7510 (2000).

[20] U. Essmann, L. Perera, M. L. Berkowitz, T. Darden, H. Lee, and L. G. Pedersen, J. Chem. Phys. 103, 8577 (1995).

[21] C. Bennemann, C. Donati, J. Baschnagel, and S. C. Glotzer, Nature 399, 246 (1999). 